\title{
Isolation of $C$. elegans and related nematodes
}

\author{
Antoine Barrière ${ }^{1}$ and Marie-Anne Félix ${ }^{2 \S}$ \\ ${ }^{1}$ Present address: Institut de Biologie du Développement de Marseille-Luminy, CNRS, France \\ ${ }^{2}$ Institut de Biologie de l'Ecole Normale Supérieure, CNRS-Inserm-ENS, Paris, France
}

\section{Table of Contents}

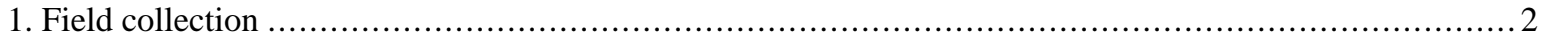

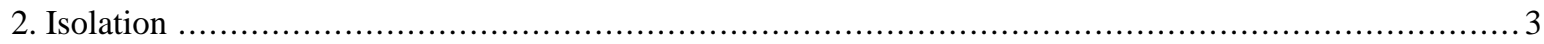

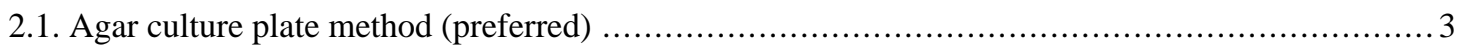

2.2. Baermann funnel ............................................................................ 3

2.3. Tray extraction (Paul De Ley, personal communication) .........................................4

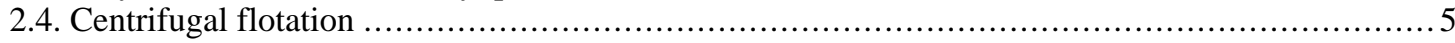

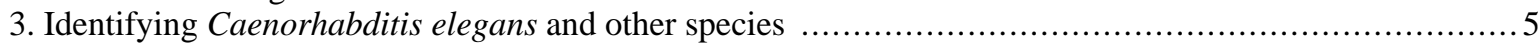

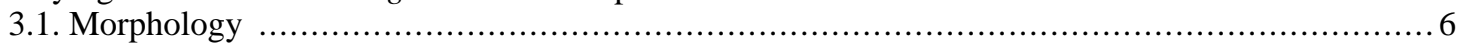

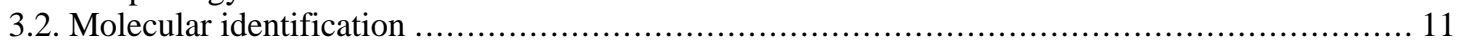

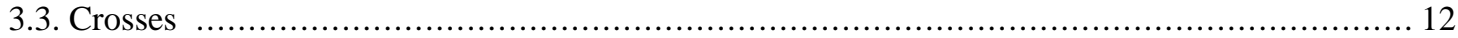

3.4. Caenorhabditis species numbering and naming ................................................. 13

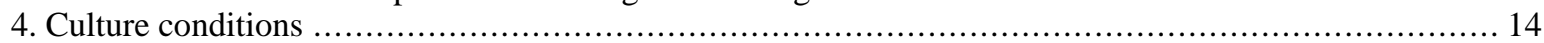

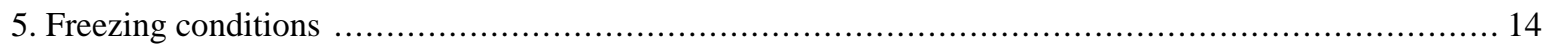

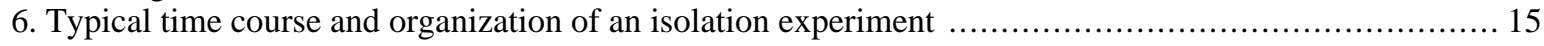

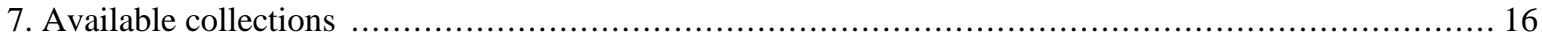

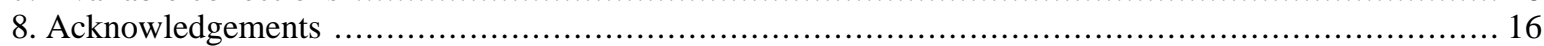

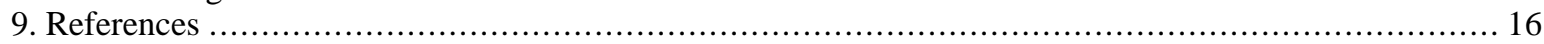

\section{Abstract}

Isolating Caenorhabditis and other nematodes from the wild first requires field sampling (reviewed in Section 1). The easiest and most efficient way to recover the animals from any substrate is to place the sample onto a standard $C$. elegans culture plate (Section 2.1). Alternative methods used by nematologists to recover soil nematodes (Sections 2.2, 2.3, and 2.4) are in our hands more difficult to implement and only yield a fraction of the individuals in the sample. A tricky step is to recognize your species of interest out of the zoo of

\footnotetext{
${ }^{*}$ Edited by Ralf Sommer. Last revised September 20, 2013. Published May 2, 2014. This chapter should be cited as: Barrière A., Félix M.-A. Isolation of C. elegans and related nematodes (May 2, 2014), WormBook, ed. The C. elegans Research Community, WormBook, doi/10.1895/wormbook.1.115.2, http://www.wormbook.org.

Copyright: () 2014 Antoine Barrière, Marie-Anne Félix. This is an open-access article distributed under the terms of the Creative Commons Attribution License, which permits unrestricted use, distribution, and reproduction in any medium, provided the original author and source are credited.

${ }^{\S}$ To whom correspondence should be addressed. E-mail: felix@biologie.ens.fr
} 
nematode species that comes with a typical sample (Section 3). Culture (Section 4) and freezing (Section 5) conditions are then reviewed. Finally, we briefly summarize the organization and timing of an isolation experiment (Section 6), as well as the available collections (Section 7). Bear in mind that this chapter is strongly focused towards the isolation of Caenorhabditis elegans and close relatives.

\section{Field collection}

Because of their size, nematodes such as $C$. elegans cannot be seen directly during sampling in the field. Collection thus proceeds blindly until return to the laboratory. The type of substrate to collect depends on the target nematode species. C. elegans was first isolated from humus, compost heaps (decaying leaves or vegetal matter) and garden soil, yet mostly in the dauer stage (Maupas, 1900; Hodgkin and Doniach, 1997; Haber et al., 2005; Barrière and Félix, 2005; Sivasundar and Hey, 2005; Cutter, 2006). Recently, large proliferating and feeding populations of various Caenorhabditis species including $C$. elegans have been found in more naturally rotting vegetal matter such as flowers and fruits decaying under their tree, and rotting stems of some herbaceous plants (Table 1) (Félix and Braendle, 2010; Kiontke et al., 2011; Félix and Duveau, 2012; Félix et al., 2013). Caenorhabditis species have only been rarely found in soil, leaf litter (including rotting grass) and rotting wood, so unless for reasons of detailed survey designs, such samples are best avoided. Many nematode species including C. elegans are also associated with invertebrates (arthropods, molluscs, etc.), chiefly but not only during their dauer stage (cf. WormBook chapter Ecology of Caenorhabditis species; Kiontke, 1997; Sudhaus et al., 2010). Some Caenorhabditis species appear to have specialized invertebrate carriers (cf. Ecology of Caenorhabditis species), especially C. japonica (Kiontke et al., 2002; Okumura et al., 2013), C. drosophilae (Kiontke, 1997) and perhaps C. angaria (Sudhaus et al., 2010). Note that the list of possible substrates from which Caenorhabditis species can be isolated (Table 1) will certainly grow.

Table 1. Occurrence of Caenorhabditis species in different substrates.

\begin{tabular}{|l|l|}
\hline Substrates where Caenorhabditis are often found & Substrates where Caenorhabditis have rarely been found \\
\hline $\begin{array}{l}\text { rotting fruits } \\
\text { rotting flowers } \\
\text { rotting stems (especially thick stems of biannual } \\
\text { species and banana pseudostems) } \\
\text { compost } \\
\text { invertebrate carriers (slugs, snails, isopods for } C . \\
\text { elegans and C. briggsae, others for other } \\
\text { Caenorhabditis species) }\end{array}$ & $\begin{array}{l}\text { soil } \\
\text { rotting leaves } \\
\text { rotting wood }\end{array}$ \\
\hline
\end{tabular}

In sum, to collect Caenorhabditis species, generally aim for VERY rotten vegetal substrates such as fruits, large flowers, stems or compost heaps. Collect either in plastic zip bags (Figure 1A) or in plastic tubes, for a volume of 1-100 $\mathrm{ml}$ per sample (typically $20 \mathrm{ml}$ ). The only important recommendation is to keep the samples aerated, i.e., unscrew slightly the cap of tubes, or do not close the bags too well. You can keep them at least 2-3 weeks (depending on the goal of your sampling, see Section 6). Avoid overheating, and overcooling below $10^{\circ} \mathrm{C}$, unless it is the natural temperature. It is best if the samples are quite humid but not flooded in the field, nor in the bag-excess liquid upon long-term conservation in a bag can be soaked up by adding a piece of paper towel (Christian Braendle, personal communication).

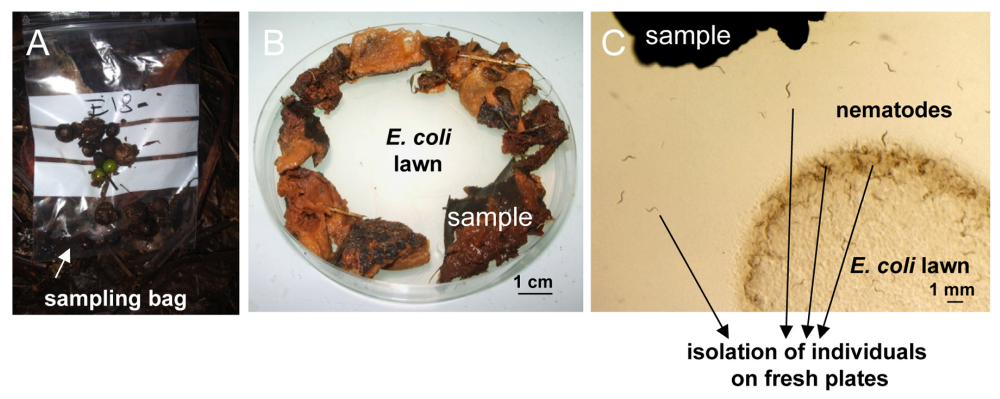

Figure 1. Nematode sampling and isolation on an agar plate. A. Sampling bag in the field. Here some rotting fruits of Euterpe sp. (palm) in Nouragues, French Guiana. B. In the laboratory, the substrate (here rotting apples) is placed around the E. coli lawn onto a standard 'C. elegans' culture plate. C. Individual nematodes that crawl out can be picked onto new culture plates. 
An alternative strategy is to use a bait to attract the nematodes, such as fruit pieces left to rot for Caenorhabditis. Worms can either move directly into the bait, or be brought through phoretic association with larger invertebrates. Fruit pieces are placed on, or in close proximity to, a substrate of interest, and collected 12 to 48 hours later. It might be necessary to protect the bait from mammals and birds with a metallic mesh. The bait is then transferred to an agar plate, and nematodes are recovered as described below.

\section{Isolation}

\subsection{Agar culture plate method (preferred)}

The easiest way to recover nematodes from a sample is simply to place the sample around the bacterial lawn of a standard C. elegans culture dish (see Maintenance of C. elegans, Section 3). Bacteria-feeding nematodes such as $C$. elegans are attracted and crawl out of the sample towards the bacterial lawn (Figure 1). For fast-moving species like C. elegans, this occurs within minutes to hours. If you want to avoid contamination by E. coli, a possibility is to attract the worms out of the sample using the attractant diacetyl.

- Spread about 1-10 grams of sample around the E. coli OP50 lawn of a standard NGM Petri dish (55 mm or 90 $\mathrm{mm}$ diameter depending on the volume of sample), and moisten with about $1 \mathrm{ml}$ M9 solution (Maintenance of $C$. elegans, Section 6, Protocol 6) or water.

- Pick to a new plate individual nematodes that have crawled out. In obligate male-female species, worms of both sexes, or a mated female, are required. Animals are ideally picked within a few hours after the sample has been placed on the plate. Later on, growing bacteria and fungi species that have been introduced with the sample may prevent worms from accessing the OP50 lawn (to avoid such growth, the sample can be moistened with $0.01 \%$ thimerosal in M9).

Using this agar plate method, all developmental stages can be recovered in a more or less quantitative manner-except for the embryonic stages, which can only be retrieved after hatching. The number of individuals in the original sample can thus be estimated (Barrière and Félix, 2005). Caenorhabditis elegans will eventually form dauers that present a characteristic waving behavior: they 'stand' on their tail on any substrate with a narrow tip (the substrate or the molds that grow out of it) and move their body, putatively as a way to make contact with and be carried away by an invertebrate (Völk, 1950; Sudhaus, 1976) and sometimes form large masses of up to thousands of individuals (Félix and Duveau, 2012). One can a posteriori check that every Caenorhabditis individual has been picked by the absence of waving dauer larvae after 6-10 days. Note that dauer larvae of other genera, such as Pristionchus, also adopt this behavior.

To recover nematodes from larger invertebrates (molluscs, arthropods, etc.), place the putative carrier onto an NGM agar plate, either alive (if you wish to test whether nematodes may disembark), naturally dead, or after sacrificing it. Wait for the nematodes to move to the OP50 lawn or collect them directly by dissection of the decaying invertebrate host.

If you target slow-growing species, a variant protocol is to place the sample onto an agar plate without nutrients (plain agar in water) and to add a chunk of NGM medium seeded with E. coli. Nematodes that like a lot of bacteria will crawl to the NGM. Species that are not so fond of extremely high bacterial density will thrive at a little distance from this chunk. This way, slowly developing species can still be isolated a little later whereas they die or get overrun by other species or fungi on NGM plates (Karin Kiontke, personal communication).

\subsection{Baermann funnel}

This is the classical method to recover a variety of nematode species, and may be particularly useful to isolate nematodes that are not attracted to E. coli, or to isolate nematodes without contamination by E. coli. Many modifications and improvements are possible, and here we describe only a single basic design.

- Fit a rubber tube onto the stem of a funnel (diameter ca. $50 \mathrm{~mm}$ ). Close the tube with a clamp (a simple paperclip can be used instead) and fill the rubber tubing with M9 solution up to the neck of the funnel (Figure 2).

- Line the funnel cone with a piece of sieve (standard cleaning tissue, muslin, mosquito mesh...), then fill with material and fold to enclose the sample. Add M9 solution or water until the sample is wet but not fully submerged.

- Active nematodes crawl through the sieve and will then sediment at the end of the tubing. Recover them by opening the clamp (from a few hours to several days later). 


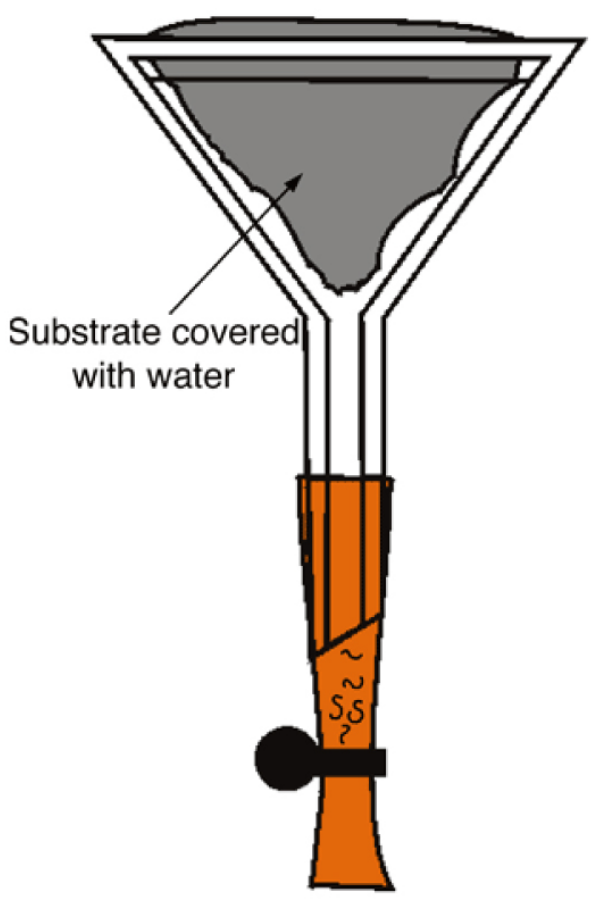

Figure 2. Baermann funnel. The nematodes swim towards the base of the funnel (this may require several days for some species; rhabditids come out after a few hours). Courtesy of Karin Kiontke.

A problem with the Baermann funnel technique, especially when isolating worms from bacteria-rich samples, is that the poor oxygenation of the sample or rapid growth of parasitic fungi will result in high nematode mortality.

\subsection{Tray extraction (Paul De Ley, personal communication)}

As with the Baermann funnel, this method is not quantitatively reliable because it selects against slow and non-moving nematodes, but it is less prone to oxygen depletion and very good at yielding large numbers of active worms from larger samples. The three components required are: (1) a large plastic tray, (2) a fitting flat mesh or strainer, and (3) sheets of strong kitchen towel paper that are larger than the mesh. The larger the tray, the more material can be extracted in one run. Do not use trays or mesh with bare metal surfaces, as these may release toxic ions.

- Place the mesh in the tray, cover its bottom and sides with one or two layers of paper, then spread a thin, uniform layer of your sample over the paper.

- Gently pour clean water into the tray and then place the mesh in the tray, making sure the sample is wet but not submerged.

- Let the tray stand for 2-12 hours, covered partly or completely to minimize evaporation. During this time, actively moving nematodes will crawl down through the paper and collect on the bottom of the tray. If the sample is very water-absorbent then check periodically underneath to ensure its soaking up of water does not leave the tray partly dry.

- After the desired time, remove the mesh with sample and pour the water out of the tray through a fine sieve (pore size $25 \mu \mathrm{m}$ or less), then wash the nematodes off the fine sieve into a small beaker with about 20-30 ml water. If you do not have a fine sieve, pour the tray water into a sufficiently large beaker and allow the nematodes to settle for about $30 \mathrm{~min}$. Pour off most of the supernatant, transfer the remainder to a smaller beaker, and repeat until you have 20-30 $\mathrm{ml}$ of concentrated nematode suspension left. 


\subsection{Centrifugal flotation}

Terrestrial nematodes have a specific gravity of 1.08 and can be recovered by flotation on a solution with a specific gravity of 1.15 (Hooper, 1986). This method is best used with soil samples, because decaying vegetal matter yields too much detritus.

Several solutions can be used:

Sucrose - is inexpensive, but sticky and has a strong osmotic effect that harms the worms $(1.71 \mathrm{~mol} / \mathrm{L}$ for a density of 1.15).

MgSO $_{4}$ - less sticky than sucrose, but also has a strong osmotic effect $(1.38 \mathrm{~mol} / \mathrm{L})$

Ludox - colloidal silica: expensive, but weak osmotic effect; samples can therefore be extracted several times, in contrast to extraction with the other solutions. Ludox comes in two forms: Ludox HS40, toxic, that may be used for fixed samples, and Ludox AS, non-toxic, that is best used to recover live material. A 50\% solution in water has a specific gravity of 1.15 .

- If your sample contains a lot of debris, rinse it first over a $1 \mathrm{~mm}$ mesh to remove the bulkiest fragments (this will however remove some of the nematodes).

- Soak the sample in water and centrifuge at $1,800 \mathrm{~g}$ to obtain a compact pellet, and eliminate the supernatant.

- Resuspend the pellet in $20 \mathrm{ml}$ of the chosen solution of density 1.15 and centrifuge at $1,800 \mathrm{~g}$ for $1 \mathrm{~min}$. Add 2 to $5 \mathrm{ml}$ of water or M9 buffer on top and centrifuge again for 5-15 min. Nematodes will accumulate at the interface between the two solutions.

- Pipet the worms at the interface (some can be seen with the naked eye) using a cut $1 \mathrm{ml}$ micropipette tip.

- Rinse them in M9, centrifuge, and pipet the pellet onto a standard NGM culture plate.

More detailed information on different isolation methods can be found in Hooper (1986) or De Ley and Mundo-Ocampo (2004).

\section{Identifying Caenorhabditis elegans and other species}

A good way to identify the genus and possibly the species is to compare observations, mostly of buccal parts and sexual organs, with published drawings or already identified cultures. For a detailed guide of soil nematodes with plenty of drawings, see Andrássy (1983, or 1984, in German); for the Rhabditidae, see the recent key in Sudhaus (2011). For the different Caenorhabditis species, see Sudhaus and Kiontke (1996) and Kiontke et al. (2011). Be aware that (1) some genera may be redefined due to new knowledge, especially molecular sequences (Scholze and Sudhaus, 2011); and (2) nematode diversity is huge and most species are still undescribed.

The increasingly used alternative to morphological identification of nematodes is based on molecular sequence tags available in databases, mostly $18 \mathrm{~S}, 28 \mathrm{~S}$ and ITS rDNA sequences. 18S rDNA is useful at a broad taxonomic level, while ITS sequences are generally useful for distinguishing conspecific strains or closely related species (Kiontke et al., 2011).

Finally, in the last step, the species identity can be determined or confirmed using crosses with cultures of known species of the genus. Modes of reproduction of different nematode species come in many flavors: male-female reproduction (as in $C$. remanei), hermaphrodite selfing with facultative male outcrossing (as in $C$. elegans), parthenogenesis (as in some Diploscapter, Panagrolaimus, Cephalobus, or Rhabditophanes spp.), partial pseudogamy (as in some Mesorhabditis spp.), and complex combinations with two possible reproductive modes-hermaphrodite selfing and male-female crosses, such as in the species related to SB347 et al. (in a yet undescribed genus) (Félix, 2004; Shakes et al., 2011). In all cases but parthenogenesis, crossing tests can be performed.

We mostly focus below on how to tell Caenorhabditis from other genera, using morphology (Section 3.1), molecular sequences (Section 3.2), and crosses with known species (Section 3.3). 


\subsection{Morphology}

The nematodes can be identified by morphological criteria either as they come out of the substrate, or perhaps more easily by observation of their progeny after they were brought into pure culture. First, most nematodes from rotting plant samples belong to one of four groups/families that can be distinguished by mouth morphology (Figure 3): Rhabditidae (including Caenorhabditis, Oscheius, Mesorhabditis, etc.), diplogastrids (Pristionchus), Panagrolaimidae (Panagrellus, Panagrolaimus), and Cephalobidae (Cephalobus, generally slower growing). Note that diplogastrids are phylogenetically nested within family Rhabditidae (Kiontke et al., 2007). See A quick tour of nematode diversity ahd the backbone of nematode phylogeny and The phylogenetic relationships of Caenorhabditis and other rhabditids for their phylogenetic relationships.

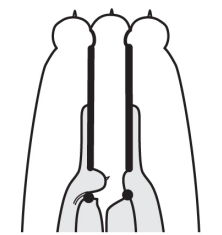

rhabditids

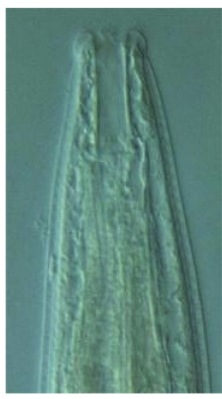

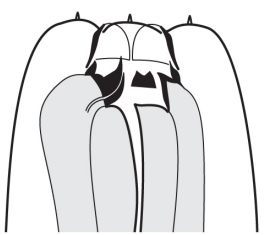

diplogastrids

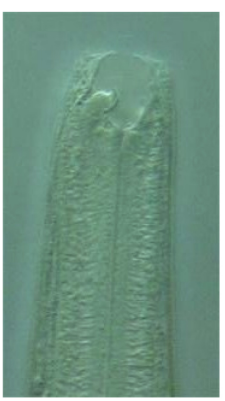

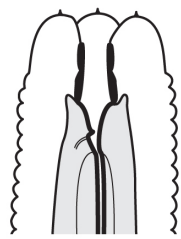

panagrolaimids

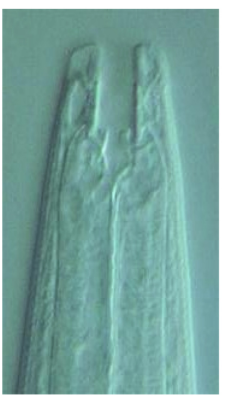

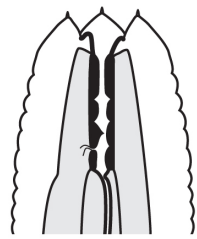

cephalobs

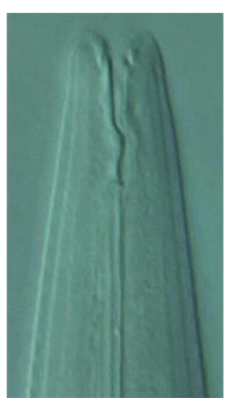

Figure 3. Schematic drawings of the characteristic buccal apparatus of different soil nematode families. Note the characteristic long and straight stoma of rhabditids, the well-developed teeth at the base of a short stoma of diplogastrids, the well-sclerotized stoma lining in panagrolaimids and the narrow stoma with a lining divided in several elements of cephalobs. Nomarski micrographs of representative nematodes of each group are shown below: Mesorhabditis longispiculosa, Pristionchus pacificus, Panagrolaimus sp., Cephalobus sp. Drawings courtesy of Karin Kiontke; pictures courtesy of Paul De Ley and Melissa Yoder.

We first review in Section 3.1.1 characteristic traits of Caenorhabditis to look for, some but not all of which are shared with other genera. For those interested, some key features of nematodes that cohabit with Caenorhabditis are presented: in Section 3.1.2 for nematodes outside the rhabditids, in Section 3.1.3 for some other rhabditid genera outside Caenorhabditis. At the species level, morphological criteria distinguishing different Caenorhabditis species are then presented in Section 3.1.4.

\subsubsection{A few traits to look for when searching for Caenorhabditis}

Here are some tips to distinguish Caenorhabditis from other genera. Some traits are most easily scored on populations, possibly cleaned by bleaching (Maintenance of C. elegans, Section 6), rather than on single individuals coming out of the sample, which may look very different because of various life adventures including pathogens. For a first triage of nematodes coming out from the sample under the dissecting microscope, look for the characters in bold.

- Caenorhabditis hermaphrodites/females generally move relatively fast and elegantly, have a long and pointy tail, which folds back on itself when the worm is moving backwards.

- Most characteristically, their gut has a homogeneous brown color in the dissecting microscope under transmitted illumination, except for few large cell nuclei, which appear white in this brown background (Figure 4). This intestinal morphological feature allows you to rule out most bacteriovorous nematodes, which have a grey or blackish intestinal color, and/or more heterogeneous coloration with a large number of small or barely visible cell nuclei (Figures 4,5, and 6). 
- The vulva is central, i.e., located towards the middle of the animal along the antero-posterior axis.

- Embryos are oval.

- The middle and basal pharyngeal bulbs (Figure 4) can both be seen with proper illumination and good magnification in the dissecting microscope. The basal bulb includes a grinder.

- When the worms are mounted and observed under a compound microscope, look for the rhabditid mouth morphology (Figure 3), the two-armed (didelphic) gonad in hermaphrodites or females and the wide cytoplasmic rachis (cf. Figure 1 in Transformation and microinjection) in the center of the dorsal gonad arms. Only Caenorhabditis sp. 1 has a single fertile arm, the anterior one (Félix and Sternberg, 1996).

- A characteristic feature of Caenorhabditis (with few exceptions) is a wide ray 6 in the male tail (see Wormatlas, Ray Composition and Structure) (Sudhaus and Kiontke, 1996).

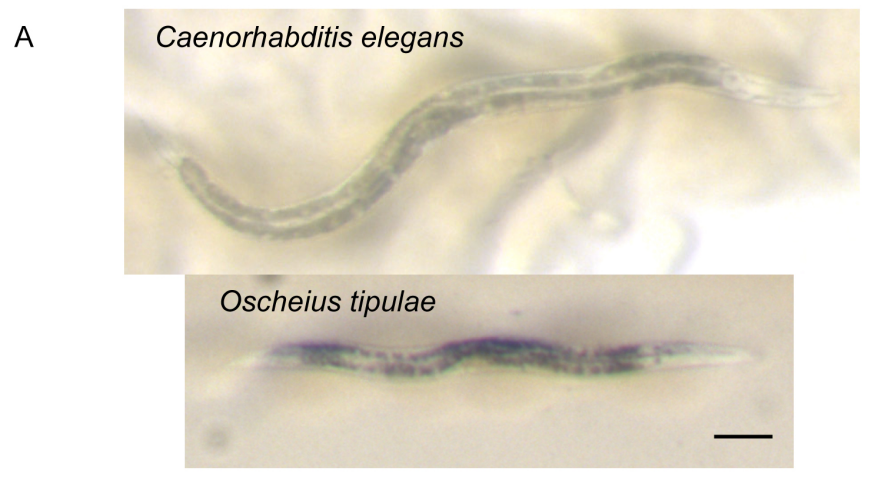

B
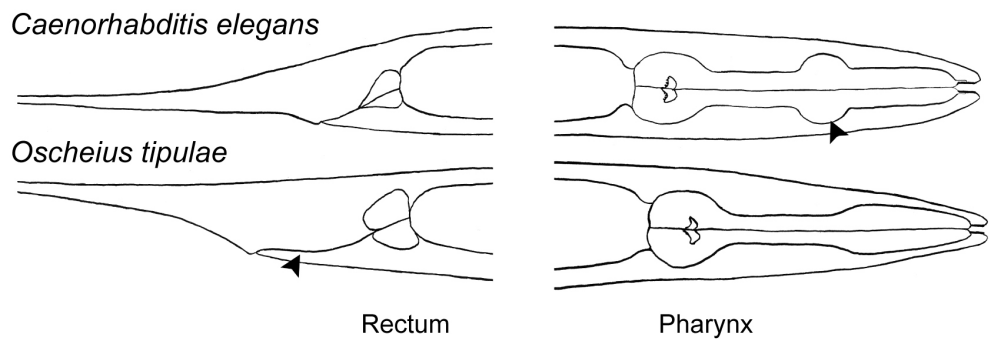

Figure 4. Distinguishing features of Caenorhabditis elegans, compared to a common hermaphroditic species, Oscheius tipulae. A. Photographs of adult hermaphrodites of Caenorhabditis elegans (top) and Oscheius tipulae (bottom). Note the light brown color of the intestinal cell contents of Caenorhabditis compared to the darker color of the intestine of many other soil nematodes, including Oscheius tipulae. In addition, the few large intestinal cell nuclei of $C$. elegans are readily visible: compare them to the smaller nuclei of Oscheius tipulae. Notice also the smaller size of $O$. tipulae. Bar: $100 \mu$ m. B. Drawings of Caenorhabditis elegans and Oscheius tipulae head and tail regions. Note the short rectum and very discernible middle pharyngeal bulb (arrowhead) of C. elegans compared to the distinctive elongated rectum (arrowhead) and simple widening of the pharynx of $O$. tipulae.

\subsubsection{Vignettes of non-rhabditid nematodes co-habiting with Caenorhabditis}

Figure 5 presents nematodes outside rhabditids that are often encountered in the same sample types as Caenorhabditis, mostly of the Panagrolaimidae and "Diplogastridae" families, but not solely. 


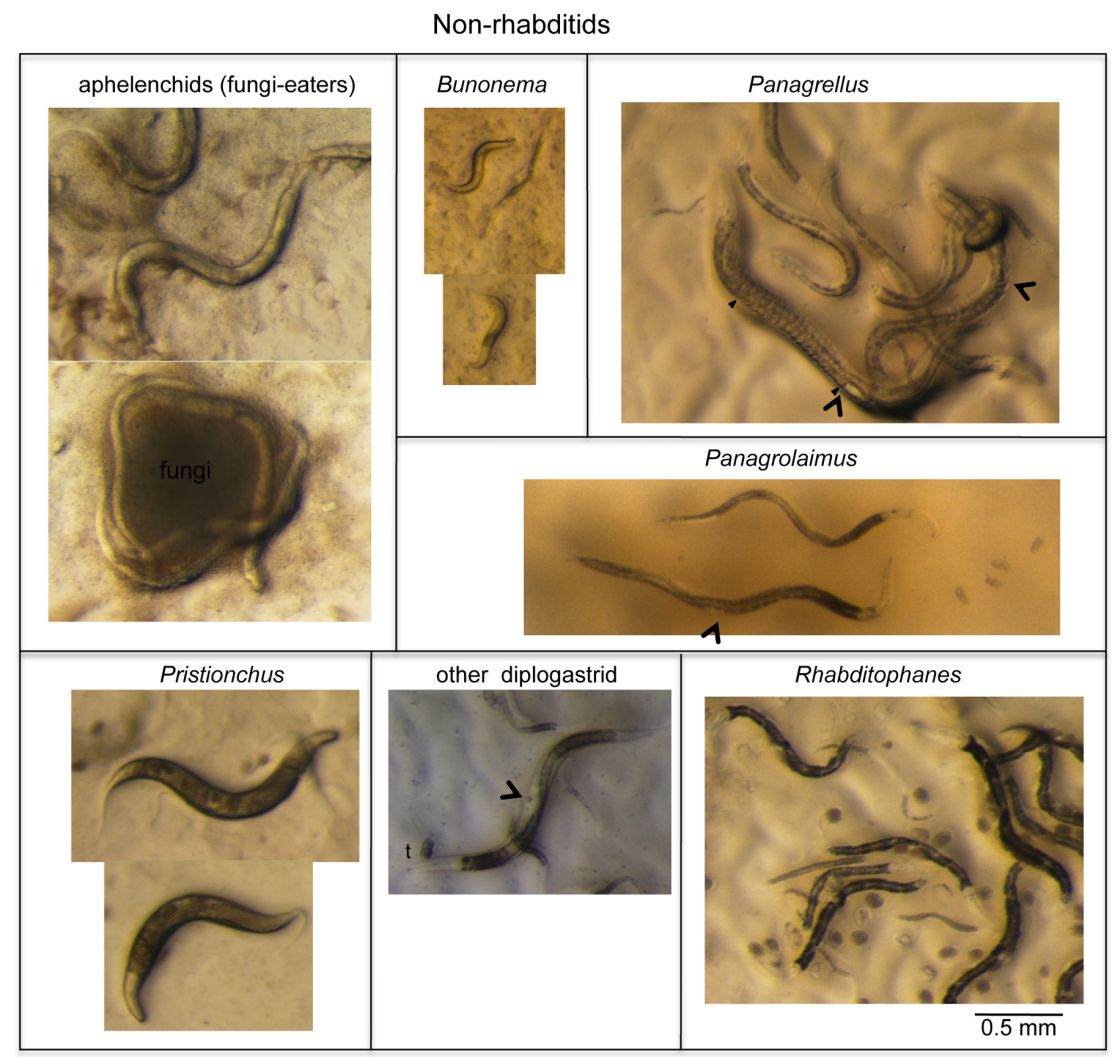

Figure 5. A few remarkable and common worms that are easy to distinguish from Caenorhabditis (part I: non-rhabditids). See Section 3.1.2. for comments. Arrowheads denote vulva positions. The 't' above the diplogastrid mouth indicates the strongly cuticularized and massive teeth of diplogastrids. Laid embryos are visible in some panels as oval-shape objects on the E. coli lawn. In the Panagrellus panel, the filled arrowhead denotes the position in the female where embryos start to accumulate up to the vulva, which is labeled with an empty arrowhead.

The Panagrolaimidae family includes a number of genera, at least three of which are readily found in rotting fruit or stem samples. Particularly frequent and specific to rotting fruits (but not stems) are Panagrellus spp. These large worms share with Caenorhabditis a brownish intestinal color and finely tapering tail under the dissecting microscope, yet (1) their vulva is quite posterior, (2) their gonad includes a single anterior ovary arm and a large post-vulval sac on the posterior side of the vulva, (3) their uterus contains all embryonic stages up to hatching as they lay their progeny as small L1 larvae, and (4) the pharynx lacks a median bulb (Figure 5) (Sternberg and Horvitz, 1981). Related to them are Panagrolaimus spp., found in a variety of substrates including dry ones. Like Panagrellus spp., Panagrolaimus spp. display a single ovary arm, yet are thinner and darker; their vulva is located almost in the middle of the animal; and they usually do not retain more than one or two embryos in their uterus and lay early-stage embryos. Finally, members of the Rhabditophanes genus, commonly found in cool meteorological conditions, are recognizable by a black intestine, roundish embryos, and fast growth (2-3 days) at $15-20^{\circ} \mathrm{C}$.

Most Diplogastridae found together with Caenorhabditis are first recognizable by their scary teeth (Figure 3). Among them, Pristionchus spp. can be recognized by strong lines all over the adult cuticle and a generally dumpyish body morphology (Figure 5). Also, diplogastrid species lack the grinder in the terminal pharynx bulb.

Two groups of slow-growing nematodes are generally only spotted several days after the samples have been placed on plates, if nothing else bloomed: (1) cephalobs are found in many environments including dry ones, and are generally below $1 \mathrm{~mm}$ in length; and (2) Bunonema nematodes are particularly small (0.3-0.4 mm; Figure 5), and easily recognizable under the compound microscope by the left-right asymmetry of their cuticular pattern and their peculiar behavior with water films (they do not wet well and float).

Fungi eaters called aphelenchids are found in fungi-rich samples, are not attracted to the $E$. coli lawn and have a pale intestine (Figure 5). In addition, one can sometimes find plant parasitic nematodes, which are very long nematodes with a stylet in the mouth that helps them allows them to puncture plant cells and ingest their contents. 


\subsubsection{Vignettes of other rhabditid genera co-habiting with Caenorhabditis}

In the Rhabditidae, a variety of genera may be found in the same samples as Caenorhabditis. Unlike Caenorhabditis, most of them show a grey or black intestinal color (Figure 6). To distinguish different rhabditid genera, we like to use vulval precursor cell lineages as observed under Nomarski optics in the early L4 stage (Kiontke et al., 2007), yet admit that this requires specialized training. Some species can instead be distinguished by a short or blunt female tail (not shown) or a posterior vulva (Figure 6) (Mesorhabditis, Teratorhabditis, Cruznema). The group of species related to SB347 (Figure 6) is common in rotting fruits and stems and may display three sexes in culture (Félix, 2004; Shakes et al., 2011), as well as tube waving behavior of the dauers, which it shares with close relatives such as Rhabditella spp.: the dauer remains inside the L2d cuticle, can exit at the front end and wave perpendicularly, yet also may retract in its shelter tube.

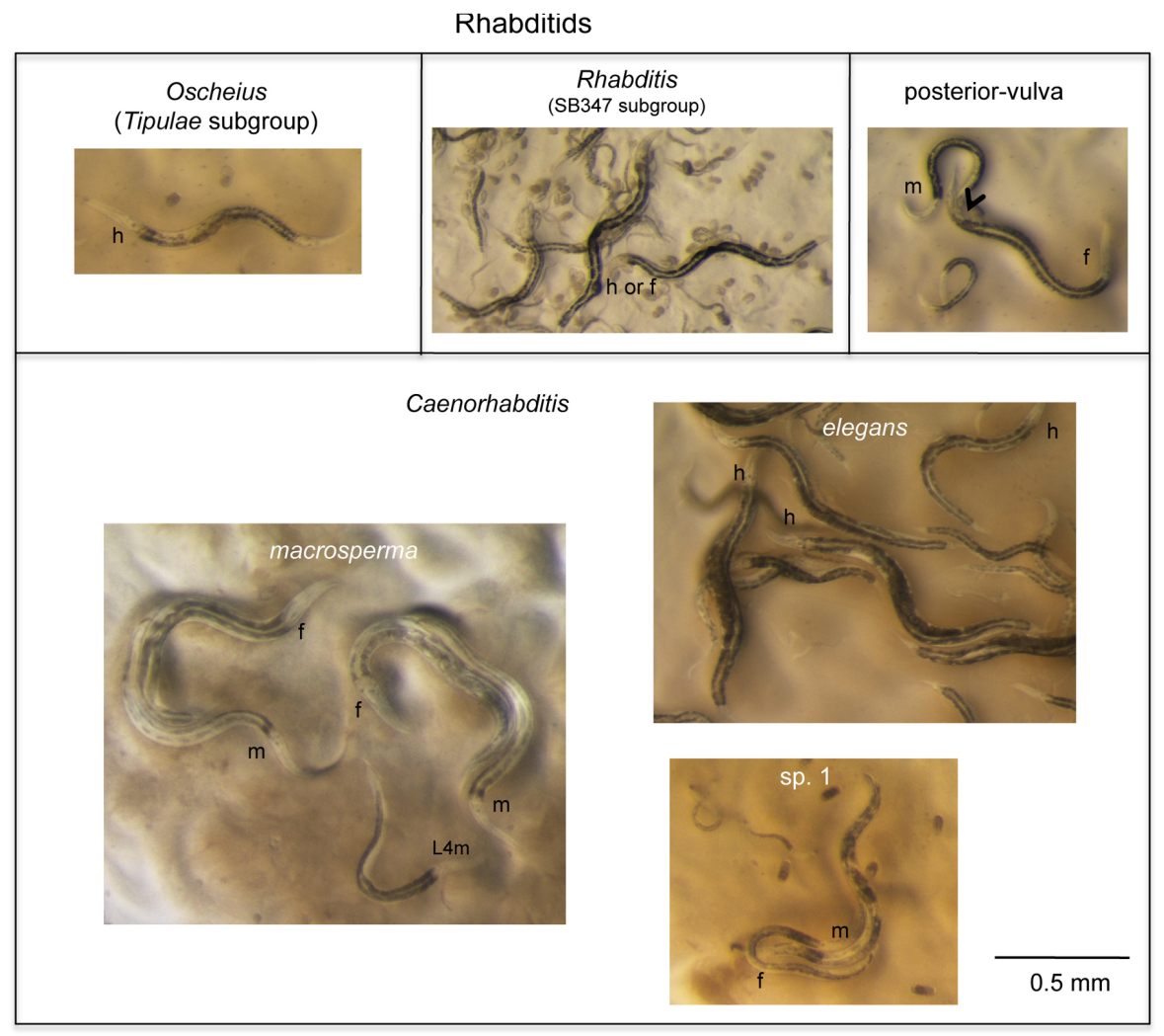

Figure 6. A few remarkable and common worms that are easy to distinguish from Caenorhabditis (part II: rhabditids). See Section 3.1.3. for comments. The ' $\mathrm{m}$ ', ' $\mathrm{f}$ ' or ' $\mathrm{h}$ ' near the basal pharyngeal bulb of an individual indicates its sex: male, female, or hermaphrodite, respectively. In the upper-right panel, the arrowhead denotes vulva position (the male is mating and an embryo has just been laid).

Most common in terrestrial habitats are Oscheius spp. The Oscheius genus is divided into two clear clades: (1) the Dolichura clade (to which $O$. tipulae belongs), with quite small animals; and (2) the Insectivora clade with animals that grow large during adulthood $(2-3 \mathrm{~mm})$ and often stop laying embryos in late life, resulting in bagging (Kiontke et al., 2007). Oscheius tipulae (see Oscheius tipulae) and close relatives are very common and are selfers like $C$. elegans. They should be easily distinguished from Caenorhabditis by their smaller size, darker and more spotted intestinal color, smaller intestinal nuclei, lack of middle pharyngeal bulb, and elongated rectum (Figure 4).

Finally, the sister group to Caenorhabditis is composed of small, slow-growing animals such as Diploscapter and Protorhabditis spp., which often move slowly through the agar of the culture plate, leaving brownish contorted trails. Protorhabditis and Prodontorhabditis species show a jerking backwards movement when disturbed (Karin Kiontke, personal communication). Diploscapter is unusual in having refringent labial hooks that are visible with a good dissection microscope (Paul De Ley, personal communication). 
Caenorhabditis animals in the dauer stage are difficult to recognize by the above criteria-so let the culture bloom from the dauer(s). However, if you see waving dauers, singly or in large masses (Félix and Duveau, 2012), they may be Caenorhabditis; note that many other species also adopt this behavior.

\subsubsection{Morphological characteristics of Caenorhabditis species}

Caenorhabditis spp. overall look similar to each other in the dissecting microscope (see Figure 6 for three examples). Indeed, most criteria mentioned below (except the spiral mating position) require mounting and observing the animals in the compound microscope, preferrably using Nomarski optics. We use these traits to narrow down of possibilities within Caenorhabditis to prioritize the crosses to known species. If these morphological criteria appear difficult to you, use molecular tags (Section 3.2).

The Caenorhabditis genus includes two large supergroups, Elegans and Drosophilae (Kiontke et al., 2011), and a few basally branching species such as $C$. plicata and $C$. sp. 1 . The latter is a small species with a single fertile ovary arm (Figure 6) (Félix and Sternberg, 1996). Defining derived morphological criteria that are diagnostic for the Elegans and Drosophilae super-groups is presently difficult. Indeed, when mapped onto the present molecular phylogeny, most morphological characters that vary in the genus show patterns of convergence and reversion (Kiontke et al., 2011). A good character seems to be the closer position of rays 2 and 3, which appears derived in the branch leading to the Elegans super-group (Kiontke et al., 2011). In contrast, finding a character such as a dorsal ray 4 (instead of a dorsal ray 5), the absence of a hook, a fan that is open on the anterior side, a short spiculum, or a spiral mating position so far excludes the possibility that the species is in the Elegans super-group and assigns them to a basal branch or to the Drosophilae super-group.

The Drosophilae super-group indeed harbors most behavioral and morphological diversity. A subclade including $C$. angaria and $C$. sp. 8 displays spiral mating (as panagrolaims and diplogastrids may also do; see Panagrellus in Figure 4), whereas all other Caenorhabditis mate in a parallel orientation as C. elegans does (see Male mating behavior). Refer to Figures 6 and 5 in Kiontke et al. (2011) for the combination of male tail morphological traits (hook and fan morphology, respective positions of rays) of different species, up to $C$. sp. 20; or to http://128.122.60.136/fmi/iwp/cgi?-db=RhabditinaDB\&-loadframes which will be updated on a regular basis with the newest species. These traits may narrow down possibilities for species identity, which is useful to prioritize crosses with known species (Section 3.3). However, be prepared and enjoy the fact that puzzling combinations of characters or new traits are still being found in the newest Caenorhabditis species (Marie-Anne Félix, Karin Kiontke and Lise Frézal, unpublished data).

In the Elegans super-group, species are very similar morphologically and in some cases cannot be distinguished from each other by specialists (e.g., Sudhaus and Kiontke, 2007). Here the mode of reproduction may provide a useful indication. So far, only three Caenorhabditis species, all of the Elegans super-group, are selfing hermaphrodites, namely C. elegans, briggsae, and tropicalis, while all other Caenorhabditis species known so far reproduce through mating of males and females. $C$. elegans and $C$. briggsae co-occur in the world regions we sample most (France). After having performed numerous diagnostic PCRs (Section 3.2) and crosses (Section 3.3), we learnt to distinguish them by several morphological and behavioral features. Specifically, C. briggsae animals are thinner and paler, lay eggs at an earlier stage and do not clump as C. elegans do (Figure 7). These features are only clear for a pure strain culture descended from one wild-caught animal and cleaned from microbial contaminants other than E. coli OP50. 


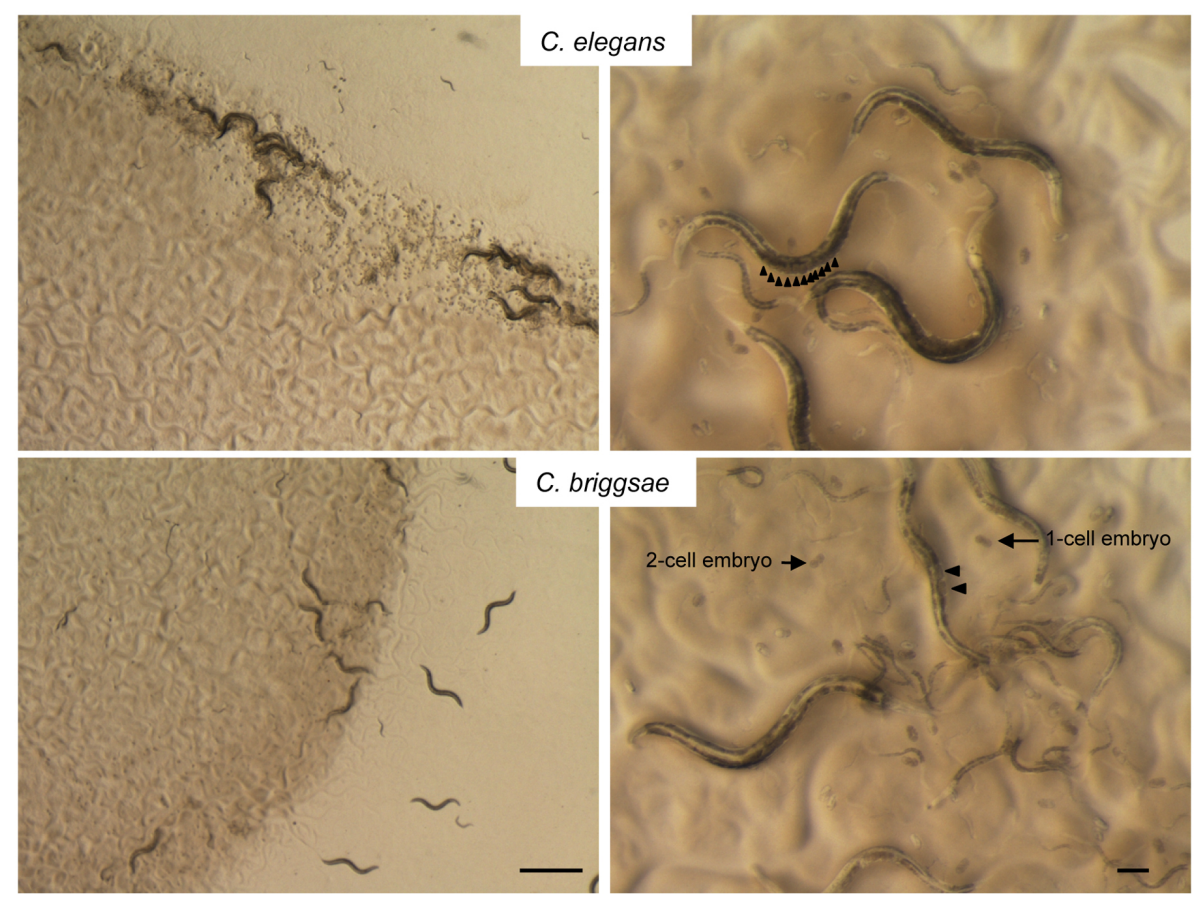

Figure 7. Distinguishing Caenorhabditis elegans from $\boldsymbol{C}$. briggsae. The following are morphological indications to distinguish the two species on a pure culture, but these cues do not replace a molecular or crossing test. Left panels: all $C$. elegans wild isolates (top) clump and border at the edge of the $E$. coli lawn, whereas C. briggsae isolates (bottom) do not clump nor border. Right panels: C. elegans isolates tend to accumulate many embryos (arrowheads) in their uterus before laying, and lay embryos at $>10$-cell stage. Note that some wild $C$. elegans isolates lay their eggs earlier than N2 and that most, unlike $\mathrm{N} 2$, clump and congregate at the edge of the E. coli lawn; also consider that young adults may not have accumulated many embryos yet. C. briggsae isolates all lay very early-stage embryos (one or two-cell stage) and generally do not have more than two fertilized embryos in their uterus under standard laboratory conditions. C. briggsae also generally looks thinner and somewhat paler than C. elegans. The third known hermaphroditic species, the tropical C. tropicalis is even thinner than C. briggsae. Bars of left panels: $1 \mathrm{~mm}$; of right panels: $0.1 \mathrm{~mm}$.

Unless one can guess which crosses to prioritize due to biogeographical species distribution (Kiontke et al., 2011; Félix and Duveau, 2012), going through a molecular sequencing tag (Section 3.2) is now advisable for species of the Elegans super-group.

\subsection{Molecular identification}

Different gene fragments are preferrably used for different taxonomic levels.

\subsubsection{Placement within nematodes: $18 \mathrm{~S}$ rDNA sequence}

Sequencing of the 18S ribosomal RNA (small subunit) gene with nematode-specific primers (Floyd et al., 2002; Haber et al., 2005) is a good way to identify the group to which a nematode belongs by BLASTing it against sequence databases. Possible primers are:

1. SSU18A (5'-AAAGATTAAGCCATGCATG)

and SSU26R (5'-CATTCTTGGCAAATGCTTTCG),

which amplify a $1 \mathrm{~kb}$ fragment of $18 \mathrm{~S}$ rDNA in rhabditid nematodes (Floyd et al., 2002), or

2. RHAB1350F (5'-TACAATGGAAGGCAGCAGGC) and RHAB1868R (5'-CCTCTGACTTTCGTTCTTGATTAA),

which amplify a fragment of about 500 bp (Haber et al., 2005), or

3. G18S4a: GCTCAAGTAAAAGATTAAGCCATGC (F) and DF18S-8: GTTTACGGTCAGAACTASGGCGG (R), which amplify a fragment of around $1 \mathrm{~kb}$ (Kiontke et al., 2004) in Rhabditidae, Diplogastridae, Panagrolaimidae, and Cephalobidae. Sequence with either PCR primer or with KK18S-8S: CGGTATCTAATCGCCTTCG (R). 


\subsubsection{Placement within Caenorhabditis: ITS2 sequence of rDNA}

At a smaller phylogenetic scale, the ITS2 region (Internally Transcribed Spacer) between the 5.8S and 28S rDNA genes provides a convenient species tag within Caenorhabditis (Kiontke et al., 2011). Possible primers are:

\subsection{S-1 (5'-CTGCGTTACTTACCACGAATTGCARAC)} and KK28S-4 (5'-GCGGTATTTGCTACTACCAYYAMGATCTGC),

which amplify a fragment of ca. $2 \mathrm{~kb}$. Sequence the fragment using the forward PCR primer and the sequencing primer KK-28S-22 (5-CACTTTCAAGCAACCCGAC).

Overall, this fragment is a useful species tag because of the significant divergence among known species (20 nucleotides or many more) and the low polymorphism within a given species (one or two single-nucleotide polymorphisms). The fact that some species (such as $C$. sp. 8) display more than a couple of nucleotide polymorphisms precludes a clear-cut new species prediction with this marker (Kiontke et al., 2011). However, once a Caenorhabditis culture is identified, the ITS2 sequence is a great test to narrow down possible species or species group.

A convenient method (Lise Frézal, personal communication) to compare a new sequence to the known ones makes use of the "Align two or more sequences" of NCBI Blast. First, retrieve known Caenorhabditis ITS2 sequences from the additional file S6 of Kiontke et al. (2011) or at http://www.ncbi.nlm.nih.gov/nuccore/ ?term=barcodes+for+Caenorhabditis+AND+ITS2. Prepare a file with these ITS2 sequences in fasta format. Blast your new sequence using the "Align two or more sequences" option of NCBI/ BLAST/ blastn. You can thus find the closest species. You can also visualize the results using the distance tree, but be aware that a tree solely based on ITS2 is poorly supported (Kiontke et al., 2011). The results indicate whether the ITS2 is identical or close to a known species.

An alternative method is to use the Rhabditina DB database by Walter Sudhaus, David Fitch, and Karin Kiontke at http://128.122.60.136/fmi/iwp/cgi?-db=RhabditinaDB\&-loadframes where you can BLAST against known rDNA sequences (even unpublished ones) using "BLAST search" in the menu on the left.

\subsubsection{Species-specific oligonucleotides: e.g., C. elegans versus $C$. briggsae}

In order to discriminate between $C$. elegans and C. briggsae (or a few locally-known species), one can perform a PCR using species-specific oligonucleotides (Barrière and Félix, 2005). For example, the following species-specific PCR primers in the $g l p-1$ gene amplify different fragment sizes in the two target species. PCR can be performed using all four primers in the same reaction (annealing $\mathrm{T}^{\circ}$ of $60^{\circ} \mathrm{C}$ ).

C. elegans: CCGCTTGGATTCTATGGATTG and CTCTCCTTGCTCCGGGATTG, product: 208 bp in $C$. elegans; none in C. briggsae.

C. briggsae: GAACCTGCGAGTGCATGTAC and CCGTCTGCAAACGAACGGGC, product: 302 bp in $C$. briggsae; none in C. elegans.

We have also successfully used other primer pairs that were available in the lab and generally only matched one species.

\subsubsection{Other genera}

Different Oscheius species of the Oscheius tipulae group can be distinguished using a PCR-RFLP test (Félix et al., 2001).

Different Pristionchus species have been distinguished by sequencing of 18S RNA (Herrmann et al., 2006).

\subsection{Crosses}

The biological species is defined and bounded by the ability to give fertile progeny in a cross. With the increasing number of Caenorhabditis species (>30 in culture), identifying a species solely based on crosses becomes cumbersome if no educated guess can be made. However, once the possibilities have been narrowed down using morphology and/or ITS sequence data, crosses are the methods of choice to formally identify a species or prove the 
discovery of a new species within a sub-group of Caenorhabditis (Félix et al., 2014). Before starting the test crosses, make sure your culture contains a single species by deriving it from a single individual or male-female pair.

Species identity can be tested by crossing several virgin (L4 stage) females or hermaphrodites of the new culture with males of identified laboratory strains, and vice versa. See Section 7 for collections from which you can obtain various species; most are at the Caenorhabditis Genetics Center (CGC), except for the last discovered species. About 5 animals of each sex are placed onto a culture plate and the culture observed over several days. In female-male species, the presence of fertile progeny of both sexes indicates that the new wild isolate is of the same species as the tester strain. In hermaphrodite-male species, successful crosses can be scored by the occurrence of a high proportion of male progeny (Nigon and Dougherty, 1949). A refined method is to use males of laboratory strains that express dominant markers (e.g., GFP), currently available for only a few species including the three selfers. In this case, cross-progeny are identified by the presence of the marker in the F1 generation and the cross-progeny may be left to reproduce to test for its fertility. Crosses between some closely related species (Baird, 2002; Sudhaus and Kiontke, 2007; Kiontke et al., 2011) may yield a few (sterile) larval and adult F1 progeny, but most of the eggs that are laid in these crosses never hatch.

As the phylogenetic density of Caenorhabditis species increases, the biological species tests are no longer clearcut: a whole continuum of cross-sterility is found. Clear instances of different species are: absence of embryonic progeny, absence of larval progeny, sterile F1 adults (often only females, Haldane's rule), or F1 adults that are fully sterile with sibs or either parent (for example C. briggsae x C. nigoni; Woodruff et al., 2010). However, in some cases, the sterility may be partial, and the species boundary becomes fuzzy, with a continuum of partial sterility phenomena. For example, crosses between some pairs of C. elegans isolates (e.g., N2 and CB4856) present 25\% dead F2 progeny, yet the causal locus for this phenomenon still recombines with the rest of the genomic diversity (Seidel et al., 2008), so here a single species should be considered. Other examples of outbreeding depression are found in C. elegans (Dolgin et al., 2007), C. briggsae (Baird and Stonisefer, 2012), and C. tropicalis (Gimond et al., 2013). At the other end of the fuzzy zone, the cross of $C$. remanei and C. latens yields hybrid F1 progeny that give very few descendants when crossed to each other (Dey et al., 2012). Note that although problematic for systematics, such situations are treasures for studying the first steps of speciation.

No progeny of $C$. elegans with any other species has been reported so far. Finding a sister species to $C$. elegans (http://forums.wormbase.org/index.php?topic=308.0; Dolgin, 2007), whether partially hybridizing with it or not, is still an unattained grail. $C$. elegans is indeed presently alone on a phylogenetic branch that goes back to the divergence with the $C$. briggsae / C. remanei /C. brenneri group. The molecular divergence of $C$. elegans with species in this clade is as high as that of mouse to human. This distance is highly problematic for ancestral state inferences, because of saturation of neutral nucleotide site turnover (Stein et al., 2003; Kiontke et al., 2004). Finding a sister species to C. elegans would break this long branch and thus be very precious for ancestral state inference and the use of C. elegans in evolutionary studies. Please sample!

\subsection{Caenorhabditis species numbering and naming}

New species are transiently ascribed a number so that one can refer to them unambiguously. Karin Kiontke acts as the unofficial curator of species numbers. If you think you may have a new Caenorhabditis species, please write to her to check on this and request a number. The latest species can be seen at http://evolution.wormbase.org/index.php/LIST_OF_AVAILABLE_CAENORHABDITIS_SPECIES_and_the_state _of_their_genome_projects. Rhabditina DB also updates morphological and molecular information about yet unpublished species (http://128.122.60.136/fmi/iwp/cgi?-db=RhabditinaDB\&-loadframes).

The actual Latin naming of these species lagged far behind because of the requirements for formal nematode species description according to zoological systematics guidelines (measurements, drawings, morphological characterization and comparison with previously descriptions; e.g., Sudhaus et al., 2010). A recent article (Félix et al., 2014) argues for the use of crosses as the defining and diagnostic character of the biological species in Caenorhabditis, a group of species where crosses are readily performed, and where taxonomic morphological characters may be polymorphic within a species, as well as conserved between species. Based on crosses, Félix et al. (2014) thus named 15 Caenorhabditis species. 


\section{Culture conditions}

When starting a culture, it is important to keep track of the number and developmental stage of the founders.

- For selfing species, the culture may be started from a single animal born in the wild and kept potentially genetically heterogeneous by the absence of further bottlenecks. This is important if studies of the natural outcrossing rate are planned. A small but not negligible proportion of wild animals are the products of recent crosses (Barrière and Félix, 2005; Sivasundar and Hey, 2005; Barrière and Félix, 2007). Thus, to obtain a genetically pure and stable isogenic line (mutation notwithstanding), it is advisable to self it by transferring a single virgin hermaphrodite animal for ca. 4-5 generations.

- For male-female species, the culture is generally started from a single mated female or a virgin female and a male, yielding an isofemale line. The culture remains genetically heterogeneous unless an isogenic line is derived by inbreeding through many generations of brother-sister mating. Note that a culture from a mated female or hermaphrodite may carry more than four alleles in its progeny since the mother may have been mated multiple times. It is thus best for diversity studies to keep track of the developmental stage of the individual that was used to start the culture. Alternatively, a strain of a male-female species can be started from several wild-born animals, yielding a population strain that minimizes inbreeding and the associated depression, and may later be a good starting material to derive inbred lines.

Many species of the Rhabditidae, Diplogastridae, Panagrolaimidae and Cephalobidae families can be grown on standard NGM plates seeded with E. coli OP50 (see Maintenance of C. elegans). Beware that many tropical Caenorhabditis species do not tolerate a storage temperature of $15^{\circ} \mathrm{C}$ and grow well at $30^{\circ} \mathrm{C}$, whereas temperate ones grow at $15^{\circ} \mathrm{C}$ but are sterile at $30^{\circ} \mathrm{C}$ (Kiontke et al., 2011). As a rule, keep temperate species at $20^{\circ} \mathrm{C}$, tropical ones at $23-25^{\circ} \mathrm{C}$.

The cultures can be cleaned of associated micro-organisms using the bleaching procedure (Maintenance of $C$. elegans, Section 6) - possibly associated with serial transfers for problematic bacteria that tend to resist this treatment. Note however that (1) associated micro-organisms may be of interest, and (2) some of these nematodes do not grow well or even enter dauer if cultured with E. coli only, and are thus more easily cultured on standard NGM plates with carried-along bacteria.

Some species of other families require special culturing media or conditions (De Ley and Mundo-Ocampo, 2004). Bunonema, Tylocephalus and Plectus can be best cultured on soil plates ( $1 \%$ agar $+1 \%$ soil $+5 \mu \mathrm{g} / \mathrm{ml}$ cholesterol), and Teratocephalus on water plates (1\% agar in water $+5 \mu \mathrm{g} / \mathrm{ml}$ cholesterol). Add ca. $2 \mathrm{ml}$ of M9 solution to the Plectus and Teratocephalus cultures (they like to swim). Keep cultures at $15-20^{\circ} \mathrm{C}$, except for Bunonema, which grows best at $25^{\circ} \mathrm{C}$.

Male-female species are generally subject to inbreeding depression, which can be very strong in some cases. This is best avoided by taking a large number of worms (>10) at each transfer and/or culturing them on larger (90 $\mathrm{mm}$ diameter) culture plates for larger population sizes.

Security proceedures: Be aware that some nematodes that can be found in soil samples such as Halicephalobus (Shadduck et al., 1979) or Pelodera (Ginsburg et al., 1984; Tanaka et al., 2004) have been described to be potentially harmful to humans. However, the major danger you may encounter is to contaminate your lab with wild mites, either nematophagous ones, swallowing worms like spaghettis, or bacterivorous ones, competing with nematodes and messing up cultures by transferring eggs and larvae between plates. Good practice is keeping your sampling plates apart from your culture plates, and wrapping them with tight Parafilm. Mites can be killed by heating plates/incubators to $50-60^{\circ} \mathrm{C}$ for a few hours; alternatively, dichlorobenzene moth balls have been reported to kill mites but not nematodes (Karin Kiontke, personal communication).

\section{Freezing conditions}

Many species can be frozen using the C. elegans freezing protocol (Maintenance of C. elegans, Section 7). However, several species do not survive freezing using this method.

For species of the Mesorhabditis, Teratorhabditis, Pelodera, Poikilolaimus, Oscheius (Insectivora group) and Panagrolaimus genera, recovery may be greatly increased if $1 \mathrm{mM} \mathrm{CaCl}_{2}$ is added to the freezing solution (Pristionchus pacificus protocols, Section 2). Adding a heat-shock treatment (e.g., $1 \mathrm{hr}$ at $37^{\circ} \mathrm{C}$ ) considerably helps with some difficult species, such as Caenorhabditis angaria or "Rhabditis" sp. SB347 and their close relatives. 
Finally, species of the Cephalobidae family are particularly drought-resistant and can be frozen by directly placing large chunks of a half-dried culture plate (slightly before it turns into an agar 'chip') into a tube at $-80^{\circ} \mathrm{C}$; for 'revival', $\mathrm{H}_{2} \mathrm{O}$ or M9 solution is added to the tube for a few hours and the tube contents are then transferred to a new culture plate.

\section{Typical time course and organization of an isolation experiment}

When sampling, keep track of geographical coordinates (GPS), date, nature of landscape, nature of substrate, so as to be able to fill in information such as found in Wormbase, e.g., http://www.wormbase.org/species /c_sp.\%2011/strain/JU1848?query=JU1848\#02-10. Try to recognize your worms of interest (see an example in Figure 8). When starting the culture, also keep track of the number, sex and stage of individuals the culture was started from.
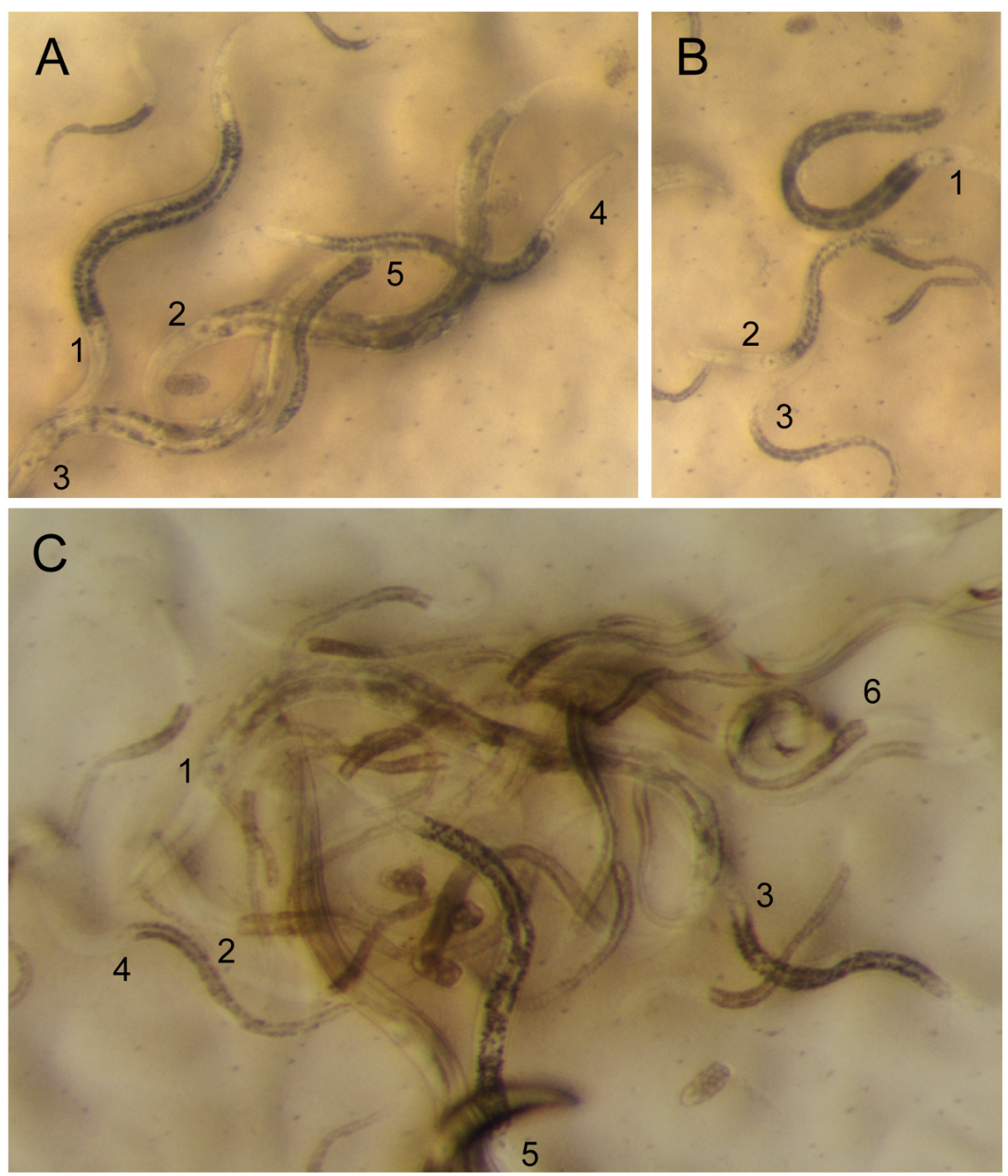

Figure 8. The real situation: find your worm. Numbers are placed next to the base of the pharynx of the animal. A. 1,4: Oscheius (tipulae or closely related species) and 2,3. Caenorhabditis adults. B. 1. Caenorhabditis L4 larva (hermaphrodite or female). 2: Oscheius L4 larva. 3. Caenorhabditis larva at the exit from the dauer stage. C. Close-up of nematodes freshly exiting the rotten apple in Figure 1C. 1: Caenorhabditis (briggsae) adult. 2. Caenorhabditis L4 larva. 3. Oscheius L4 larva. 4. Caenorhabditis L3, likely postdauer, larva. 5. Oscheius adult. 6. Three diplogastrid males are visible on the top right. These diplogastrids do not have a grinder at the base of the pharynx. The fast-running worm next to \#5 is likely a diplogastrid L4 larva.

The incidence of Caenorhabditis in wet tropical areas is high, and half of the samples of rotting fruits, flowers and stems may yield a Caenorhabditis species, sometimes several (Félix et al., 2013). However, the incidence of Caenorhabditis in temperate areas appears much lower, and even in a location where Caenorhabditis is found, only one in ten samples may be positive (Félix and Duveau, 2012). 
The organization of a nematode isolation experiment depends on its goal:

1. If the goal is to study wild worms to directly determine their number, sex, stage, associated flora, etc., the worms need to be isolated within minutes to a few hours after sample collection.

2. If the goal is to obtain an accurate representation of genetic diversity of a natural population, one needs to isolate the individuals that were present at the time of sampling, that is, within a single generation (obviously, the time between sample isolation and plating must be reduced to the minimum).

3. If the goal is to keep a maximum of one genotype of each species per sample, the sample can be plated several days after its collection (up to several months for some drought-resistant nematodes). Specifically, Caenorhabditis can be recovered after up to three weeks of sample collection, yet most likely with a much reduced efficiency compared to fresh samples. Do not hesitate to pool several flowers or small fruits or several pieces of different fruits at the time of sampling in the same bag, then keep a single isolate per species per sample.

\section{Available collections}

- Caenorhabditis Genetics Center: http://www.cbs.umn.edu/CGC/strains/

- Many wild isolates are listed in Wormbase under «Strain » with their sampling details and lab of origin, e.g., http://www.wormbase.org/species/c_elegans/strain/JU2001?query=JU2001\#02-10

Individual labs:

- Félix lab collection: http://www.justbio.com/worms/index.php

- Braendle lab collection: http://www.braendlelab.net/worms.html

- Cutter lab database: http://labs.eeb.utoronto.ca/cutter/map/ (includes isolates from the above labs).

- Fitch lab collection (especially for Rhabditidae outside Caenorhabditis): http://www.nyu.edu/projects/fitch /Rhabditina/NYURC_strainslist.htm

- Sommer lab collection (Pristionchus): http://www.pristionchus.org/cgi-bin/strain_req.pl

- See also http://evolution.wormbase.org/index.php/Main_Page for news on Caenorhabditis species, especially on sampling and species discovery

\section{Acknowledgements}

We are grateful to Karin Kiontke and Paul De Ley for providing drawings, pictures and protocols. We thank Asher Cutter, Lise Frézal, Christian Braendle, Karin Kiontke and the reviewers for helpful comments.

\section{References}

Andrássy, I. (1983). A taxonomic review of the suborder Rhabditina (Nematoda:Secernentia) (Paris: ORSTOM).

Andrássy, I. (1984). Klasse Nematoda (Ordnungegen Monhysterida, Desmoscolecida, Araeolaimida, Chromadorida, Rhabditida) (Stuttgart: Gustav Fischer Verlag).

Baird, S.E. (2002). Haldane's rule by sexual transformation in Caenorhabditis. Genetics 161, 1349-1353. Abstract

Baird, S.E., and Stonisefer, R. (2012). Reproductive isolation in Caenorhabditis briggsae: Dysgenic interactions between maternal- and zygotic-effect loci result in a delayed development phenotype. Worm 1, 189-195. Abstract

Barrière, A., and Félix, M.A. (2005). High local genetic diversity and low outcrossing rate in Caenorhabditis elegans natural populations. Curr. Biol. 15, 1176-1184. Abstract Article 
Barrière, A., and Félix, M.A. (2007). Temporal dynamics and linkage disequilibrium in natural Caenorhabditis elegans populations. Genetics 176, 999-1011. Abstract Article

Cutter, A.D. (2006). Nucleotide polymorphism and linkage disequilibrium in wild populations of the partial selfer Caenorhabditis elegans. Genetics 172, 171-184. Abstract Article

De Ley, P., and Mundo-Ocampo, M. (2004). Cultivation of Nematodes. In Nematology-Advances and Perspectives Volume 1, Z. Chen, S. Chen, and D.W. Dickson, eds. (CABI publishing, Tsinghua University Press), pp. 541-619. Abstract

Dey, A., Jeon, Y., Wang, G.X., and Cutter, A.D. (2012). Global population genetic structure of Caenorhabditis remanei reveals incipient speciation. Genetics 191, 1257-1269. Abstract Article

Dolgin, E.S., Charlesworth, B., Baird, S.E., and Cutter, A.D. (2007). Inbreeding and outbreeding depression in Caenorhabditis nematodes. Evolution 61, 1339-1352. Abstract Article

Dolgin, E. (2007). Slime for a dime. Science 317, 1157. DOI: 10.1126/science.317.5842.1157b Abstract Article

Félix, M.-A. (2004). Alternative morphs and plasticity of vulval development in a rhabditid nematode species. Dev. Genes Evol. 214, 55-63. Abstract Article

Félix, M.-A., and Braendle, C. (2010). The natural history of Caenorhabditis elegans. Curr. Biol. 20, R965-R969. Abstract Article

Félix, M.-A., and Sternberg, P.W. (1996). Symmetry breakage in the development of one-armed gonads in nematodes. Development 122, 2129-2142. Abstract

Félix, M.-A., Braendle, C., Cutter, A.D. (2014). A streamlined system for species diagnosis in Caenorhabditis (Nematoda: Rhabditidae) with name designations for 15 distinct biological species. PLoS One, 9, e94723. Abstract Article

Félix, M.-A., Vierstraete, A., and Vanfleteren, J. (2001). Three biological species related to Rhabditis (Oscheius) pseudodolichura Körner in Osche, 1952. J. Nematol. 33, 104-109. Abstract

Félix, M.A., and Duveau, F. (2012). Population dynamics and habitat sharing of natural populations of Caenorhabditis elegans and C. briggsae. BMC Biol. 10, 59. Abstract Article

Félix, M.A., Jovelin, R., Ferrari, C., Han, S., Cho, Y.R., Andersen, E.C., Cutter, A.D., and Braendle, C. (2013). Species richness, distribution and genetic diversity of Caenorhabditis nematodes in a remote tropical rainforest. BMC Evol. Biol. 13, 10. Abstract Article

Floyd, R., Abebe, E., Papert, A., and Blaxter, M. (2002). Molecular barcodes for soil nematode identification. Mo. Ecol. 11, 839-850. Abstract Article

Gimond, C., Jovelin, R., Han, S., Ferrari, C., Cutter, A.D., and Braendle, C. (2013). Outbreeding depression with low genetic variation in selfing Caenorhabditis nematodes. Evolution 67, 3087-3101. Abstract Article

Ginsburg, B., Beaver, P.C., Wilson, E.R., and Whitley, R.J. (1984). Dermatitis due to larvae of a soil nematode, Pelodera strongyloides. Pediatr. Dermatol. 2, 33-37. Abstract Article

Haber, M., Schungel, M., Putz, A., Muller, S., Hasert, B., and Schulenburg, H. (2005). Evolutionary history of Caenorhabditis elegans inferred from microsatellites: evidence for spatial and temporal genetic differentiation and the occurrence of outbreeding. Mol. Biol. Evol. 22, 160-173. Abstract Article

Herrmann, M., Mayer, W.E., and Sommer, R.J. (2006). Nematodes of the genus Pristionchus are closely associated with scarab beetles and the colorado potato beetle in Western Europe. Zoology 109, 96-108. Abstract Article

Hodgkin, J., and Doniach, T. (1997). Natural variation and copulatory plug formation in Caenorhabditis elegans. Genetics 146, 149-164. Abstract 
Hooper, D.J. (1986). Extraction of free-living stages from soil. In Laboratory methods for work with plant and soil nematodes, J.F. Southey, ed. (London: HMSO), pp. 5-30.

Kiontke, K. (1997). Description of Rhabditis (Caenorhabditis) drosophilae n. sp. and R. (C.) sonorae n. sp. (Nematoda: Rhabditida) from saguaro cactus rot in Arizona. Fund. Appl. Nematol. 20, 305-315. Abstract

Kiontke, K., Barrière, A., Kolotuev, I., Podbilewicz, B., Sommer, R.J., Fitch, D.H.A., and Félix, M.-A. (2007). Trends, stasis and drift in the evolution of nematode vulva development. Curr. Biol. 17, 1925-1937. Abstract

Kiontke, K., Gavin, N.P., Raynes, Y., Roehrig, C., Piano, F., and Fitch, D.H. (2004). Caenorhabditis phylogeny predicts convergence of hermaphroditism and extensive intron loss. Proc Natl Acad Sci USA 101, 9003-9008. Abstract

Kiontke, K., Hironaka, M., and Sudhaus, W. (2002). Description of Caenorhabditis japonica n. sp. (Nematoda: Rhabditida) associated with the burrower bug Parastrachia japonensis (Heteroptera: Cynidae) in Japan. Nematology 4, 933-941. Abstract

Kiontke, K., Félix, M.-A., Ailion, M., Rockman, M.V., Braendle, C., Pénigault, J.-B., and Fitch, D.H. (2011). A phylogeny and molecular barcodes for Caenorhabditis, with numerous new species from rotting fruits. BMC Evol. Biol. 11, 339. Abstract Article

Maupas, E. (1900). Modes et formes de reproduction des nématodes. Arch. Zool. Exp. Gen. 8, 463-624. Abstract

Nigon, V., and Dougherty, E.C. (1949). Reproductive patterns and attempts at reciprocal crossing of Rhabditis elegans Maupas, 1900, and Rhabditis briggsae Dougherty and Nigon, 1949 (Nematoda: Rhabditidae). J. Exp. Zool. 112, 485-503. Abstract Article

Okumura, E., Tanaka, R., and Yoshiga, T. (2013). Species-specific recognition of the carrier insect by dauer larvae of the nematode Caenorhabditis japonica. J. Exp. Biol. 216, 568-572. Abstract Article

Scholze, V.S. and Sudhaus, W. (2011). A pictorial key to current genus groups of "Rhabditidae". J. Nematode Morphol. Syst. 14, 105-112. Abstract

Seidel, H.S., Rockman, M.V., and Kruglyak, L. (2008). Widespread genetic incompatibility in C. elegans maintained by balancing selection. Science 319, 589-594. Abstract Article

Shadduck, J.A., Ubelaker, J., and Telford, V.Q. (1979). Micronema deletrix meningoencephalitis in an adult man. Am. J. Clin. Pathol. 72, 640-643. Abstract

Shakes, D.C., Neva, B.J., Huynh, H., Chaudhuri, J., and Pires-Dasilva, A. (2011). Asymmetric spermatocyte division as a mechanism for controlling sex ratios. Nat. Commun. 2, 157. Abstract Article

Sivasundar, A., and Hey, J. (2005). Sampling from natural populations with RNAI reveals high outcrossing and population structure in Caenorhabditis elegans. Curr. Biol. 15, 1598-1602. Abstract Article

Stein, L.D., Bao, Z., Blasiar, D., Blumenthal, T., Brent, M.R., Chen, N., Chinwalla, A., Clarke, L., Clee, C., Coghlan, A., et al. (2003). The genome sequence of Caenorhabditis briggsae: a platform for comparative genomics. PLoS Biol. 1, 166-192. Abstract Article

Sternberg, P.W., and Horvitz, H.R. (1981). Gonadal cell lineages of the nematode Panagrellus redivivus and implications for evolution by the modification of cell lineage. Dev. Biol. 88, 147-166. Abstract Article

Sudhaus, W. (1976). Vergleichende Untersuchungen zur Phylogenie, Systematik, Ökologie, Biologie und Ethologie der Rhabditidae (Nematoda). Zoologica 43, 1-228. Abstract

Sudhaus, W. (2011). Phylogenetic systematisation and catalogue of paraphyletic "Rhabditidae" (Secernentea, Nematoda). J. Nematode Morphol. Syst. 14, 113-178. 
Sudhaus, W., and Fitch, D. (2001). Comparative studies on the phylogeny and systematics of the Rhabditidae (Nematoda). J. Nematol. 33, 1-70.

Sudhaus, W., and Kiontke, K. (1996). Phylogeny of Rhabditis subgenus Caenorhabditis (Rhabditidae, Nematoda). J. Zool. Sys. Evol. Res. 34, 217-233. Abstract Article

Sudhaus, W., and Kiontke, K. (2007). Comparison of the cryptic nematode species Caenorhabditis brenneri sp. n. and $C$. remanei (Nematoda: Rhabditidae) with the stem species pattern of the Caenorhabditis Elegans group. Zootoxa $1456,45-62$.

Sudhaus, W., Kiontke, K., and Giblin-Davis, R.M. (2010). Description of Caenorhabditis angaria n. sp. (Nematoda: Rhabditidae), an associate of sugarcane and palm weevils (Coleoptera: Curculionidae). Nematology 13, 61-78.

Tanaka, A., Kinoshita, M., Tanaka, T., Iwanaga, Y., Kagei, N., and Hide, M. (2004). Pelodera strongyloides infestation presenting as pruritic dermatitis. J. Am. Acad. Dermatol. 51, S181-184. Abstract Article

Völk, J. (1950). Die Nematoden der Regenwürmer und aasbesuchenden Käfer. Zool. Jb Syst. 79, 1-70.

Woodruff, G.C., Eke, O., Baird, S.E., Félix, M.-A., and Haag, E.S. (2010). Insights into species divergence and the evolution of hermaphroditism from fertile interspecies hybrids of Caenorhabditis nematodes. Genetics 186, 997-1012. Abstract

All WormBook content, except where otherwise noted, is licensed under a Creative SOMRERIGHISRESERVED Commons Attribution License. 